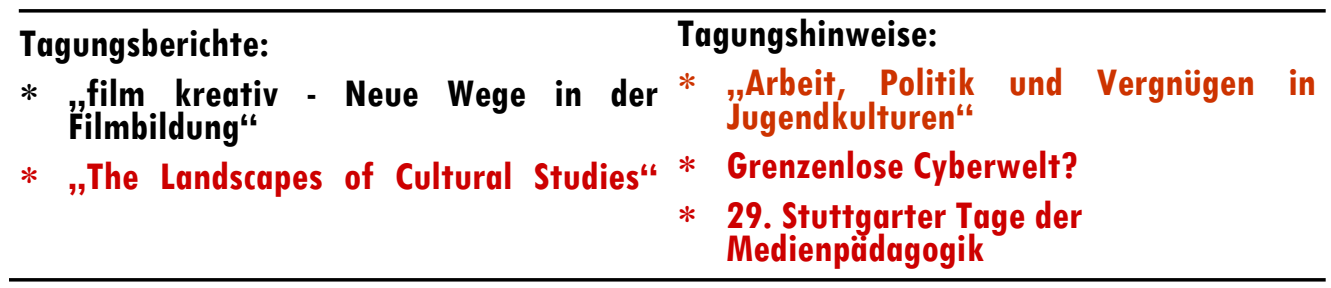

film kreativ - neue Wege in der schulischen und außerschulischen Filmbildung

Bundesweite Fachtagung mit 140 Teilnehmern an der Pädagogischen Hochschule Ludwigsburg am 2./3. Dezember 2005

\section{HORST NIESYTO}

Filme sind nicht nur Unterrichtsmedien Filme sind vor allem Ausdrucks- und Kommunikationsmedien mit vielfältigen Möglichkeiten. Obgleich Filme und audiovisuelle Medien in der heutigen Zeit eine enorme Rolle in Beruf und Freizeit spielen, mangelt es nach wie vor an einer breiten Filmbildung in der Schule und anderen Bildungsbereichen. Kinder und Jugendliche verfügen zwar über Film- und Kinowissen; es fehlen jedoch genaue Kenntnisse über die Codes, die Gestaltungs- und die Manipulationsmöglichkeiten von Filmen. ,Lesen ' heißt gerade in unserer Mediengesellschaft nicht nur Lesen von Texten und Büchern, sondern auch Verstehen von Filmen, von visuellen und audio-visuellen Medienangeboten.

Die Tagung an der PH Ludwigsburg ging vor allem der Frage nach, was die dringendsten Aufgaben zur Verbesserung der Filmbildung sind. Eingeladen hatten das Interdisziplinäre Zentrum für Medienpädagogik und Medienforschung (IZMM) an der Pädagogischen Hochschule, das Landesmedienzentrum (LMZ) und das Evangelische Medienhaus Stuttgart. Hanns-Georg Helwerth, Roland Kohm und die Professoren Peter Imort (Musik) und Horst Niesyto (Abteilung Medienpädagogik) hatten mit Unterstützung der Landesanstalt für Kommunikation (LfK, Stuttgart) ein vielfältiges Programm vorbereitet. Mit Plenumsvorträgen, Workshops, Podiumsgesprächen und auf einem „Markt der Möglichkeiten“ wurden vorhandene Initiativen zur Filmbildung vorgestellt und neue Aufgaben benannt. Reinhard Middel von der Bundesinitiative „Vision Kino“ (Potsdam) sagte, dass sich im Bereich der Lehrerfortbildung, der lokalen Vernetzung von Fachkräften und der Methoden und Arbeitsformen einiges verändern müsse. Björn Maurer, Lehrer an einer
Ludwigsburger Grundschule und PHMedienpädagoge, gab einen eindrucksvollen Einblick in kreative Methoden der Filmbildung: am vorhandenen Film- und Medienwissen von Kindern und Jugendlichen ansetzen, spielerisch vorgehen, gestalterische Aufgabenstellungen und Übungen integrieren, die Schüler/innen als Persönlichkeiten stärken.

Die Tagungsteilnehmer/innen waren sich darin einig, dass künftige Filmbildung noch besser auf die Bedürfnisse unterschiedlicher Zielgruppen eingehen muss. Gefragt sind anschauliche, konkrete Materialien und Arbeitshilfen, eine bessere Verankerung der Filmbildung in der gesamten Lehrerbildung und erheblich mehr Anstrengungen im Bereich der Lehrerfortbildung sowie in der Kooperation von schulischen und außerschulischen Initiativen. Vielleicht gelingt es im Raum Ludwigsburg / Stuttgart ein regionales Filmkompetenzzentrum aufzubauen, um insbesondere im Fortbildungsbereich Fortschritte zu erzielen. Hans Beerstecher von der Landesanstalt für Kommunikation (LfK) sagte, dass er ein solches Vorhaben sehr unterstützenswert fände. 


\section{Tagungsbericht „The Landsca- pes of Cultural Studies"}

Alpen-Adria Cultural Studies Konferenz an der Universität Klagenfurt, 13.-15. Oktober 2005

\section{Peter HOLZWARTH}

Vom 13.10. - 15.10.2005 wurde an der AlpenAdria Universität in Klagenfurt am Wörther See die Konferenz „The Landscapes of Cultural Studies" abgehalten. Rainer Winter und seinem engagierten Organisationsteam ist eine höchst spannende, vielseitige und internationale Veranstaltung zu verdanken.
Thematische Vorschläge für die Tagung waren „hybride Identitäten“, „borderlands“, „Diaspora“, „Ethnizität und Konflikt" sowie spezielle Aspekte des Alpen-Adria-Raums. Das Themenspektrum der Beiträge war breit und vielseitig: z. B. Die Krise der Kultur und alternative Formen von Modernität (Lawrence Grossberg), Kritik an George W. Busch (Douglas Kellner), Nationale und rechte Symbole im deutschen HipHop (Udo Göttlich), Der Weltjugendtag im Kontext von Globalisierung, Netzwerken der Medien transkultureller Kommunikation/ http://www.wjt-forschung.de (Andreas Hepp): Migration und Mediennutzung in der Schweiz (Heinz Moser), Whiteness (Catarina Fox), Kriegsberichterstattung (Horst Tonn), Ge-

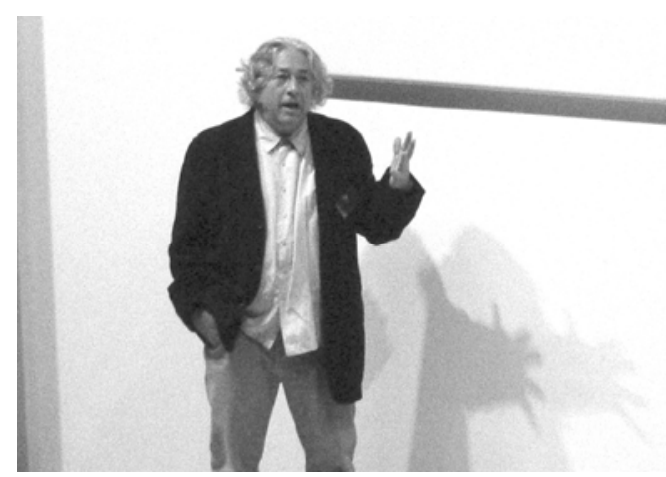

Lawrence Grossberg und Rainer Winter

Forscherinnen und Forscher aus 19 Ländern und 4 Kontinenten (Österreich, Deutschland, der Schweiz, Slowenien, Italien, Großbritannien, USA, Polen, Kroatien, Kanada, den Niederlanden, Lettland, Portugal, Türkei, Indonesien, Finnland, Belgien, Taiwan und Sambia) präsentierten und diskutierten Theorien, Konzepte, Projekte und Visionen. Auffällig viele junge Forscherinnen und Forscher waren vertreten.

Das globale Projekt der Cultural Studies, das sich durch die Aspekte Kontextorientierung, Selbstreflexivität, Interdisziplinarität und Orientierung an sozialer Veränderung auszeichnet, vertritt einen dynamischen, pluralen, prozesshaften, semiotischen und antiessentialistischen Kulturbegriff. schlecht und Medien (u. a. Brigitte Hipfel, Miriam Strube), Kulturspezifische Umgangsweisen mit Höflichkeit und direkte vs. Indirekte Kommunikation im Ländervergleich (Iris Wangerman). Entwicklungsperspektiven der Cultural Studies (Hanno Hardt), Gilles Deleuze und die Cultural Studies (Olaf Sanders), Das do-ityourself-Prinzip in der Jugendkultur Hardcore (Marc Calmbach), Mediale Aneignungsformen (Sven Tiermann), Hybridität, Sprache und Übersetzung (Andreas Hetzel), Der Herr der Ringe (u. a. Lothar Mikos), Mehrfachzugehörigkeit junger Migranten im Kontext globaler, lokaler und herkunftsbezogener medialer Ressourcen (Peter Holzwarth), u. v. a.

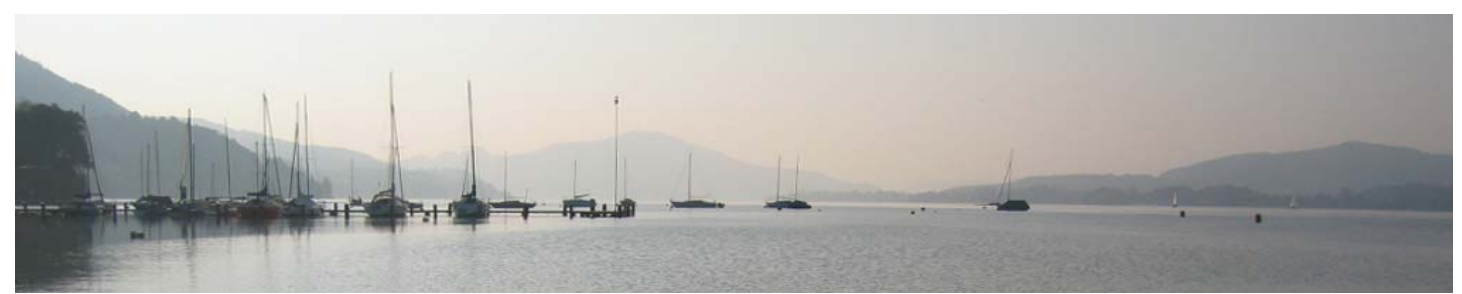

Der Wörther See, ca. 12 Minuten von der Universität Klagenfurt entfernt 
In verschiedenen Beiträgen ging es auch immer wieder um das Selbstverständnis der Cultural Studies, deren Status und institutionelle Verortung. Auch die Rolle des Forschers/der Forscherin wurde diskutiert (verschiedene Grade von "going native“, verschiedene Grade von sozialer und politischer Motiviertheit).

Methodologische und methodische Fragestellungen standen in den Beiträgen insgesamt weniger stark im Zentrum.

Douglas Kellner bezog sich in seinem Beitrag explizit auf die Arbeiten des marxistischen Philosophen Ernst Bloch, mit dem er selbst bei einem Studienaufenthalt in Tübingen in Kontakt war. Im Kontext von „media manipulation“ in den USA wies er auch auf die wichtige Bedeutung von Media literacy und Medienpädagogik hin.

\section{Internetseiten}

Internetseite zur Tagung:

http://www.uni-klu.ac.at/mk/CS2005/

Tagungsprogramm:

http:/ / www.uni-klu.ac.at/mk/CS2005/doc/csprogramm-031005.pdf

Homepage Institut für Medien- und Kommunikationswissenschaft:

http://www.uni-klu.ac.at/mk/

Homepage von Andreas Hepp:

http://www.andreas-hepp.name/

Page von Douglas Kellner:

http:/ /www.gseis.ucla.edu/faculty/kellner/

kellnerhtml

\section{Literaturhinweise}

Friedrich Krotz: Neue Theorien entwickeln. Eine Einführung in die Grounded Theory, die Heuristische Sozialforschung und die Ethnographie an Hand von Beispielen aus der Kommunikationsforschung. Herbert von Halem Verlag. Köln 2005

Grossberg, Laurence: Was sind Cultural Studies? In: Hörnig, Karl H. \& Winter, Rainer (Hg.): Widerspenstige Kulturen. Cultural Studies als Herausforderung. Frankfurt am Main: Suhrkamp 1999, S. 43-84

Hepp, Andreas \& Winter, Rainer: Kultur - Medien - Macht. Cultural Studies und Medienanalyse. Opladen: Westdeutscher Verlag, 2., überarbeitete Auflage 1999

Hepp, Andreas: Cultural Studies und Medienanalyse. Opladen: Westdeutscher Verlag 1999

Hepp, Andreas: Netzwerke der Medien. Medienkultur und Globalisierung. Wiesbaden: Verlag für Sozialwissenschaften 2004

Hörnig, Karl H. \& Winter, Rainer (Hg.): Widerspenstige Kulturen. Cultural Studies als Herausforderung. Frankfurt am Main: Suhrkamp 1999
O'Sullivan, Tim / Hartley, John / Saunders, Danny / Montgomery, Martin / Fiske, John: Key Concepts in Communication and Cultural Studies. London und New York: Routledge 1994 Winter, Carsten / Thomas, Tanja / Hepp, Andreas (Hg.): Medienidentitäten. Identität im Kontext von Globalisierung und Medienkultur. Köln: Halem 2003

Winter, Rainer: Der produktive Zuschauer. Medienaneignung als kultureller und ästhetischer Prozeß. München: Quintessenz 1995 


\title{
Arbeit, Politik und Vergnügen in Jugendkulturen
}

\author{
Tagung der Sektionen Jugendsoziologie und Kultursoziologie der DGS \\ in Kooperation mit dem Interdisziplinären Zentrum für Medienpädagogik und \\ Medienforschung (IZMM, PH Ludwigsburg) \\ vom 2. bis 3. März 2006 an der PH Ludwigsburg
}

\section{Tagungsankündigung}

Was normalerweise für unvereinbar gehalten wird, verbinden Jugendliche in jugendkulturellen Aktivitäten offenbar ganz selbstverständlich: Arbeit und Kreativität, Politik und Vergnügen. In einer Freizeitgesellschaft, in der ungewollte Freizeit zunimmt, bzw. in einer Arbeitsgesellschaft, in der Arbeit immer knapper wird, sieht die Jugendkulturforschung jugendkulturelles Verhalten längst nicht mehr ausschließlich als Freizeitgestaltung an. Vielmehr werden Jugendkulturen und Jugendszenen zunehmend als Ort für Professionalisierungs-, Selbstbildungs- und Selbstsozialisationsprozesse betrachtet, als Grundlage zum Erwerb (sub-)kulturellen Kapitals, das durchaus in ökonomisches Kapital transformierbar ist.

Allen Unkenrufen zum Trotz ist politische Aktivität nicht völlig aus Jugendkulturen verschwunden: Aus der Sicht ihrer Mitglieder thematisiert zum Beispiel die Jugendkultur Hardcore marginalisierte gesellschaftspolitische Fragen und motiviert zu politisch-sozialem Engagement. Politik in Jugendkulturen äußert sich auch in der Widerständigkeit gegenüber der Vereinnahmung durch den Mainstream; das Spektrum erstreckt sich von symbolischer Widerspenstigkeit bis zu realpolitischem jugendkulturellen Handeln. Symbolische Widerspenstigkeit erscheint auch in der jugendkulturellen Vereinnahmung von Religion als ästhetisches Stilmittel, wobei Religion neuerdings wieder verstärkt eine sozialintegrative und persönlichkeitsbildende Funktion $\mathrm{zu}$ übernehmen scheint.

Die Tagung nimmt Jugendkulturen zum einen aus der Perspektive der eher ,traditionellen“ Begriffe von Arbeit und Politik in den Blick nehmen, wobei produktive jugendkulturelle Tätigkeit meist nicht bereits Erwerbsarbeit darstellt, wohl aber in diese münden kann. Zum anderen betrachten Begriffe wie „Identitätsarbeit“ und „Identitätspolitiken“ jugendkulturelle Produktivität und Widerspenstigkeit aus neuen Perspektiven, beispielsweise der einer subjektorientierten Soziologie. Neuere jugendkulturelle Studien (Eckert et al., Klein \& Friedrich, Hitzler et al.) machen deutlich, dass die Teilnahme in jugendkulturellen Szenen ohne vielfältige selbst organisierte Aneignungsprozesse szenespezifischen Wissens, von Kompetenzen und Fertigkeiten nicht möglich ist. Die sozialen Bedeutungen solcher Prozesse
(Identitätskonstruktion und -präsentation, Selbstverortung im sozialen Raum, Konstruktionen von Authentizität) werden untersucht. Die Tagung nimmt unter die Lupe, wie diese Aneignungsprozesse ablaufen, wie und wodurch sie befördert und behindert werden.

Darüber hinaus stellt die Tagung auch quasi Selbstverständliches in Frage stellen: Was bedeuten eigentlich Vergnügen und Spaß in Jugendkulturen aus jugend- und kultursoziologischer Perspektive? Und wann wird aus Spaß Ernst? Welche Politik des Vergnügens lässt sich identifizieren? Im Mittelpunkt der Betrachtung unterschiedlicher Jugendkulturen, Jugendszenen und -cliquen wird die Frage danach stehen, welche Antwort sich in ihnen auf die widersprüchlichen Handlungsanforderungen in der Spätmoderne bei der Bewältigung individualisierter Lebensführung findet. Wie steht es in diesem Zusammenhang mit der Vorstellung subversiver jugendkultureller Praktiken? Wurden diese durch Lebensstile und Szenen absorbiert und finden nun einen anderen Ausdruck, oder wurden subkulturelle Praktiken schlicht verdrängt? Welche Argumente sprechen für einen Wandel von Subkulturen zu Szenen? Und schließlich, wie sieht es kulturtheoretisch mit der Frage von Integration und Desintegration aus? Inwiefern sind die kulturellen Praktiken Ausdruck der Bewältigung gesellschaftlicher Herausforderungen und welche „Kultur“ bildet sich aus? Nicht zuletzt die Frage nach dem Klassenbezug von Jugendkulturen, die im Zuge der Postmodernisierung der Jugendkulturkonzepte kaum mehr gestellt wurde, wird wieder aufgegriffen.

In fünf thematische Blöcken widmen sich dreißig Referentinnen und Referenten aus Deutschland, Österreich und der Schweiz den genannten Fragestellungen und Problembereichen:

- Kompetenzerwerb und symbolische Kreativität in Jugendkulturen,

- Jugendkulturen als politische und religiöse Sinnstiftungen,

- Sozialästhetische Umgehensweisen mit Musik und Medien in Jugendkulturen,

- Alltagskultur und institutionelle / gesellschaftliche Integration,

- Forschungszugänge zu jugendkulturellen Selbstinszenierungen.

Die Tagungswebseite findet sich unter: http://www.ph-ludwigsburg.de/3224.html 


\section{Grenzenlose Cyberwelt?}

Digitale Ungleichheit und neue Bildungszugänge für Jugendliche

Internationale Fachtagung in Bielefeld, 9. - 11. Februar 2006

Tagungsprogramm und Informationen unter http://www.kib-bielefeld.de/tagung
Durchlässig und (un-)durchschaubar. Datenschutz im digitalen Zeitalter

29. Stuttgarter Tage der Medienpädagogik,

17. / 18. März 2006 in Stuttgart-Hohenheim

Tagungsprogramm unter:

http:/ /www.lfk.de/home/29.stgt_tage_medien paedagogik180106.pdf

Anmeldung: info@akademie-rs.de 